\title{
Average-Preserving Symmetries and Equipartition in Linear Hamiltonian Systems
}

\author{
Sanjay P. Bhat \\ Dept of Aerospace Engineering \\ Indian Institute of Technology \\ Powai, Mumbai 400076, India \\ (+91-22) 2576-7142, 2572-2602 (Fax) \\ bhat@aero.iitb.ac.in
}

\begin{abstract}
This paper analyzes equipartition in linear Hamiltonian systems in a deterministic setting. We consider the group of phase space symmetries of a stable linear Hamiltonian system, and characterize the subgroup of symmetries whose elements preserve the time averages of quadratic functions along the trajectories of the system. As a corollary, we show that if the system has simple eigenvalues, then every symmetry preserves averages of quadratic functions. As an application of our results to linear undamped lumped-parameter systems, we provide a novel proof of the virial theorem using symmetry. We also show that under the assumption of distinct natural frequencies, the time-averaged energies of two identical substructures of a linear undamped structure are equal. Examples are provided to illustrate the results.
\end{abstract}

\section{Introduction}

Undamped and thus conservative mechanical systems are Lyapunov stable, and thus have no steadystate energy distribution. The simplest example of an undamped single-degree-of-freedom oscillator shows that the energy is periodically converted from purely kinetic to purely potential and then back to purely kinetic. A two-degree-of-freedom system consisting of an interconnected pair of undamped oscillators exhibits similar behavior, with the energy alternately possessed by one oscillator and then the other. Yet, in classical statistical thermodynamics, a crystalline solid is modeled as a lattice of identical vibrating molecules, whose degrees of freedom satisfy the principle of equipartition of energy. Thus, despite the lack of a steady-state energy distribution in undamped systems, such systems provide the conceptual foundation for macroscopic energy transfer when the system consists of a large number of undamped oscillators.

\author{
Dennis S. Bernstein
}

Dept. of Aerospace Engineering

The University of Michigan

Ann Arbor, MI 48109-2140

(734) 764-3719, (734) 763-0578 (Fax)

dsbaero@engin.umich.edu

The principle of equipartition of energy implies that the temperature of each subsystem converges to the same value, and leads to the zeroth law of thermodynamics, that is, that heat flows from hot to cold. The principle of equipartition holds for systems with linear dynamics; for nonlinear systems it is well known that equipartition does not generally occur [7].

The challenge of deriving the empirical laws of thermodynamics from large scale systems of discrete subsystems depends on the statistical properties of the system model. Stochastic averaging can be performed with respect to the statistics of initial conditions, exogenous disturbances, or subsystem and coupling parameters (see $[8,9,10]$ and the references therein). Whichever approach is used, the ultimate objective is to approximate the high-order dynamics of an oscillatory system with the low-order dynamics of a non-oscillatory system. In short, wave dynamics are approximated by diffusion dynamics. Related work from a dynamics and control perspective can be found in $[1,5,6,13,14]$.

The objective of this paper is to investigate equipartion in a deterministic setting in undamped systems whose number of degrees of freedom may not be large.

In [4] a deterministic averaging approach was used to analyze equipartition in collections of identical, undamped coupled oscillators. It was shown that equipartition of energy holds for a pair of identical, coupled oscillators with distinct coupled frequencies. This result shows that, with time averaging, energy flows from the initially higher-energy oscillator to the initially lowerenergy oscillator, thereby verifying the zeroth law of thermodynamics for a pair of coupled oscillators. In addition, numerical evidence was presented to suggest that the analogous result holds for a collection of coupled oscillators.

Reference [12] adopted a deterministic averaging approach in a behavioral framework to analyze equipar- 
tition in an oscillatory system comprising of two identical subsystems coupled together in a symmetrical manner. It was shown that if the coupling rendered the motion of each of the two subsystems observable through the variables of the other, then, along every motion of the coupled system, the time average of any given quadratic functional of the variables of one subsystem equals the time average of the same functional of the variables of the other subsystem. In particular, this implies that the energies of the two subsystems are equal in the average.

Intuitively, one expects that a system of coupled subsystems will exhibit equipartition of energy only if the subsystems are identical, that is, the state variables of one subsystem transform to those of the other subsystem under a state-space symmetry $S$ that leaves the dynamics of the overall coupled system unchanged. Moreover, the energies of such identical subsystems are quadratic functions on the full state space that transform into one another under the symmetry transformation $S$. Hence the average energies of the two identical subsystems are equal if the symmetry $S$ that relates the two subsystems also preserves averages of functions.

To formalize the above ideas we consider the Lie group $\mathbf{G}_{A}$ of phase space symmetries of a linear Hamiltonian system $\dot{y}=A y$. Thus $\mathbf{G}_{A}$ is the set of all symplectic transformations that leave the dynamics of the system invariant. As expected from Noether's theorem [2, Appendix 5], the Lie algebra of $\mathbf{G}_{A}$ is the set of all linear Hamiltonian systems whose Hamiltonian functions are the quadratic integrals of motion of the original system.

In section 4, we consider averages of quadratic functions along the solutions of a stable Hamiltonian system. In section 5, we identify symmetries of the Hamiltonian system that preserve averages of quadratic functions. Our main result says that the symmetries in $\mathbf{G}_{A}$ that preserve averages of every quadratic function form the subgroup $\mathfrak{G}_{A}$ of elements that are also symmetries of every linear Hamiltonian system whose Hamiltonian function is an integral of motion of the original system.

In section 6, we characterize the groups $\mathbf{G}_{A}$ and $\mathfrak{G}_{A}$ along with their Lie algebras and describe their structure in terms of the eigenstructure of the system. In particular, we show that the subgroup $\mathfrak{G}_{A}$ of averagepreserving symmetries is the center subgroup of $\mathbf{G}_{A}$ consisting of those symmetries of the system that commute with every other symmetry of the system. As a corollary of our characterization, we show that if the system has distinct eigenvalues, then $\mathfrak{G}_{A}=\mathbf{G}_{A}$, that is, every symmetry preserves averages. This corollary partly justifies the assumption of distinct coupled natural frequencies used in [4].

In section 7 , we apply our main result to undamped linear lumped-parameter systems. We show that the kinetic and potential energies of such a system, considered as quadratic functions on the phase space, are related by a phase space symmetry of the system that commutes with every other phase space symmetry. An application of our main result thus yields a novel proof of the virial theorem for linear systems, which states that the average kinetic and potential energies of an undamped linear mechanical system are equal. We also specialize our main result to configuration space symmetries, that is, orthogonal transformations on the configuration space that leave the mass and stiffness matrices invariant. We show that under the assumption of distinct natural frequencies, every configuration-level symmetry preserves the average of a quadratic function of positions or velocities. We provide an example to illustrate how this corollary can be used to deduce equipartition among identical subsystems of an undamped system. We also provide an example to demonstrate that equipartition may not hold if the assumption of distinct natural frequencies fails.

\section{Integrals of Motion and Symmetries of Linear Hamiltonian Systems}

For every $n$, we let $I_{n}$ denote the identity matrix of size $n \times n$, and let $J_{2 n}=\left[\begin{array}{cc}0 & I_{n} \\ -I_{n} & 0\end{array}\right]$. A matrix $S \in$ $\mathbb{R}^{2 n}$ is symplectic if $S^{\mathrm{T}} J_{2 n} S=J_{2 n}$, and Hamiltonian if $J_{2 n} S$ is symmetric. We denote by $\mathbf{S p}(n)$ the set of all $2 n \times 2 n$ real symplectic matrices. $\mathbf{S p}(n)$ is a $n(2 n+1)$ dimensional Lie group [11, p. 8]. The Lie algebra of $\mathbf{S p}(n)$ is the set $\mathbf{s p}(n)$ of $2 n \times 2 n$ real Hamiltonian matrices. Finally, for every $n$, we let $\operatorname{Sym}(n)$ denote the $\frac{1}{2} n(n+1)$-dimensional real vector space of all $n \times n$ real symmetric matrices.

A matrix $B \in \mathbf{s p}(n)$ is stable if every eigenvalue of $B$ is semisimple and has zero real part. A matrix $S \in$ $\mathbf{S p}(n)$ is stable if every eigenvalue of $S$ is semisimple and has unit magnitude. Every real eigenvalue of a stable symplectic matrix is thus either 1 or -1 .

In this paper, we consider the autonomous linear Hamiltonian system

$$
\dot{y}(t)=A y(t),
$$

where $y(t) \in \mathbb{R}^{2 n}$ and $A \in \mathbf{s p}(n)$. The matrix $H \stackrel{\text { def }}{=}$ $J_{2 n}^{-1} A$ is symmetric, and the system (1) is Hamiltonian with the quadratic Hamiltonian function $x \mapsto x^{\mathrm{T}} H x$. 
We define $\mathcal{L}_{A}: \operatorname{Sym}(2 n) \rightarrow \operatorname{Sym}(2 n)$ by $\mathcal{L}_{A}(Q)=$ $A^{\mathrm{T}} Q+Q A$. For every $Q \in \operatorname{Sym}(2 n)$, the quadratic function $x \mapsto x^{\mathrm{T}} \mathcal{L}_{A}(Q) x$ is the Lie derivative of the quadratic function $x \mapsto x^{\mathrm{T}} Q x$ along trajectories of (1), that is, for every solution $y$ of (1) and for every $Q \in \operatorname{Sym}(2 n)$, it follows that $\frac{d}{d t} y^{\mathrm{T}}(t) Q y(t)=$ $y^{\mathrm{T}}(t) \mathcal{L}_{A}(Q) y(t)$. Thus, for every $Q \in$ kernel $\mathcal{L}_{A}$, the quadratic function $x \mapsto x^{\mathrm{T}} Q x$ is an integral of motion for the system (1).

Next, we introduce the set $\mathbf{G}_{A} \stackrel{\text { def }}{=}\{S \in \mathbf{S p}(n)$ : $\left.S^{-1} A S=A\right\}$ of symplectic transformations with respect to which the dynamics (1) are invariant. It is easy to show that $\mathbf{G}_{A}=\left\{S \in \mathbf{S p}(n): S^{\mathrm{T}} H S=H\right\}$. $\mathbf{G}_{A}$ is clearly a group. The next result asserts that $\mathbf{G}_{A}$ is a Lie group. Furthermore, the Lie algebra of $\mathbf{G}_{A}$ is the Lie algebra of linear Hamiltonian systems whose Hamiltonian functions are precisely the quadratic integrals of motion of (1).

Proposition 2.1. $\quad \mathbf{G}_{A}$ is a closed Lie subgroup of $\mathbf{S p}(n)$ having Lie algebra $\mathbf{g}_{A}=\{B \in \mathbf{s p}(n): B A-$ $A B=0\}=\left\{J_{2 n} P: P \in\right.$ kernel $\left.\mathcal{L}_{A}\right\}$. In particular, $\mathbf{g}_{A}$ and kernel $\mathcal{L}_{A}$ have the same dimension.

Proof. It follows from Proposition I.2.1.3 of [11] that $\mathbf{G}_{A}$ is a Lie group with Lie algebra $\mathbf{g}_{A}$. The last part follows by showing that $B \in \mathbf{s p}(n)$ satisfies $B A=$ $A B$ if and only if $P \stackrel{\text { def }}{=} J_{2 n}^{-1} B \in \operatorname{kernel} \mathcal{L}_{A}$.

Remark 2.1. The relationship between the symmetries and the integrals of motion of (1) pointed out in Proposition 2.1 is a restatement of the Hamiltonian version of Noether's theorem (see, for instance, [2, Appendix 5]). However, we do not elaborate on this connection as it lies outside the scope of this paper.

\section{Time-Averages of Quadratic Functions}

In this section, we investigate time-averages of quadratic functions along the solutions of (1).

In the sequel, we assume that the Hamiltonian ma$\operatorname{trix} A$ is stable. Thus $A$ has semisimple imaginary eigenvalues, and every solution of (1) is bounded. In particular, the matrix exponential $t \mapsto e^{A t}$ is bounded.

Next, we define $\mathcal{A}: \operatorname{Sym}(2 n) \rightarrow \operatorname{Sym}(2 n)$ by $\mathcal{A}(Q)=\lim _{t \rightarrow \infty} \frac{1}{t} \int_{0}^{t} e^{A^{\mathrm{T}} \tau} Q e^{A \tau} d \tau$. For every $x \in \mathbb{R}^{2 n}$ and every $Q \in \operatorname{Sym}(2 n), x^{\mathrm{T}} \mathcal{A}(Q) x$ is the average of the quadratic function $z \mapsto z^{\mathrm{T}} Q z$ over $[0, \infty)$ along the solution $y$ of (1) satisfying $y(0)=x$. Thus kernel $\mathcal{A}$ represents quadratic functions that have zero average along trajectories of (1).
The next result relates the property of being zero mean along trajectories of (1) to the property of being an integral of motion of (1).

\section{Proposition 3.1.}

$$
\begin{aligned}
\text { range } \mathcal{A} & =\operatorname{kernel} \mathcal{L}_{A}, \\
\text { range } \mathcal{L}_{A} & =\operatorname{kernel} \mathcal{A} .
\end{aligned}
$$

Proof. Let $Q \in \operatorname{Sym}(2 n)$ and consider $P=\mathcal{A}(Q)$. We have $\mathcal{L}_{A}(P)=\lim _{t \rightarrow \infty} \frac{1}{t} \int_{0}^{t} \frac{d}{d t} e^{A^{\mathrm{T}} \tau} Q e^{A \tau} d \tau=$ $\lim _{t \rightarrow \infty} \frac{1}{t}\left[e^{A^{\mathrm{T}} t} Q e^{A t}-Q\right]=0$. Thus range $\mathcal{A} \subseteq$ kernel $\mathcal{L}_{A}$. The reverse inclusion follows by noting that $e^{A^{\mathrm{T}} \tau} Q e^{A \tau}=Q$ for every $\tau \geq 0$ and $Q \in$ kernel $\mathcal{L}_{A}$, so that the restriction of $\mathcal{A}$ to kernel $\mathcal{L}_{A}$ equals the identity. Equation (2) now follows.

On noting that $\mathcal{L}_{A} \circ \mathcal{A}=\mathcal{A} \circ \mathcal{L}_{A}$, it follows from (2) that range $\mathcal{L}_{A} \subseteq$ kernel $\mathcal{A}$. To show the reverse inclusion, we use $(2)$ to compute $\operatorname{dim}\left(\right.$ range $\left.\mathcal{L}_{A}\right)=n(2 n+$ 1) $-\operatorname{dim}\left(\operatorname{kernel} \mathcal{L}_{A}\right)=n(2 n+1)-\operatorname{dim}(\operatorname{range} \mathcal{A})=$ $\operatorname{dim}($ kernel $\mathcal{A})$. Since range $\mathcal{L}_{A}$ is contained in kernel $\mathcal{A}$ and has the same dimension as kernel $\mathcal{A},(3)$ follows.

\section{Average-Preserving Symmetries}

In this section, we identify symmetries of (1) that preserve time averages of quadratic functions along solutions of (1).

Let $\mathfrak{G}_{A}=\cap_{B \in \mathbf{g}_{A}} \mathbf{G}_{B}$ denote the set of symplectic symmetries of (1) that are also symmetries of every linear Hamiltonian system whose Hamiltonian function is an integral of motion of (1). The center subalgebra [11, p. 40] of $\mathbf{g}_{A}$ is the set $\mathfrak{g}_{A} \stackrel{\text { def }}{=}\left\{C \in \mathbf{g}_{A}: B C-C B=\right.$ $\left.0, B \in \mathbf{g}_{A}\right\}$ of elements in $\mathbf{g}_{A}$ that commute with every element of $\mathbf{g}_{A}$. The subalgebra $\mathfrak{g}_{A}$ is a commutative Lie subalgebra of $\mathbf{g}_{A}$. It is easy to show that $\mathfrak{g}_{A}=\cap_{B \in \mathbf{g}_{A}} \mathbf{g}_{B}$. Our next result states that $\mathfrak{G}_{A}$ is a Lie subgroup of $\mathbf{S p}(n)$ and has the Lie algebra $\mathfrak{g}_{A}$.

Proposition 4.1. $\mathfrak{G}_{A}$ is a Lie subgroup of $\mathbf{S p}(n)$ having the Lie algebra $\mathfrak{g}_{A}$.

Proof. The result follows from Proposition 2.1 and Theorem I.1.4.2 of [11].

The following theorem which is our main result, asserts that if two quadratic functions are related by a symmetry of (1) contained in $\mathfrak{G}_{A}$, then the two functions have the same average along solutions of (1).

Theorem 4.1. Suppose $S \in \mathbf{G}_{A}$. Then $\mathcal{A}\left(S^{\mathrm{T}} Q S\right)=\mathcal{A}(Q)$ for every $Q \in \operatorname{Sym}(2 n)$ if and only if $S \in \mathfrak{G}_{A}$.

Proof. We begin by noting that since $S e^{A t}=e^{A t} S$ for every $t$, it follows that $\mathcal{A}\left(S^{\mathrm{T}} Q S\right)=S^{\mathrm{T}} \mathcal{A}(Q) S$ for 
every $Q \in \operatorname{Sym}(2 n)$.

To show sufficiency, suppose $S \in \mathfrak{G}_{A}$ and let $Q \in \operatorname{Sym}(2 n)$. Proposition 3.1 implies that $\mathcal{A}(Q) \in$ kernel $\mathcal{L}_{A}$, so that $J \mathcal{A}(Q) \in \mathrm{g}_{A}$ by Proposition 2.1. It now follows by definition that $S \in \mathbf{G}_{J \mathcal{A}(Q)}$, that is, $S^{\mathrm{T}} \mathcal{A}(Q) S=\mathcal{A}(Q)$. Hence $\mathcal{A}\left(S^{\mathrm{T}} Q S\right)=\mathcal{A}(Q)$.

Next, to prove necessity, suppose $\mathcal{A}\left(S^{\mathrm{T}} Q S\right)=$ $\mathcal{A}(Q)$ for every $Q \in \operatorname{Sym}(2 n)$, and let $B \in \mathbf{g}_{A}$. Define $Q \stackrel{\text { def }}{=} J_{2 n}^{-1} B \in \operatorname{Sym}(2 n)$. Proposition $2.1 \mathrm{im}$ plies that $Q \in$ kernel $\mathcal{L}_{A}$, so that $\mathcal{A}(Q)=Q$. Hence $S^{\mathrm{T}} Q S=S^{\mathrm{T}} \mathcal{A}(Q) S=\mathcal{A}\left(S^{\mathrm{T}} Q S\right)=\mathcal{A}(Q)=Q$, which implies that $S B=B S$, that is, $S \in \mathbf{G}_{B}$. Since $B \in \mathbf{g}_{A}$ was chosen arbitrarily, it follows that $S \in \mathfrak{G}_{A}$.

\section{Structure of the Groups $\mathbf{G}_{A}$ and $\mathfrak{G}_{A}$}

In this section, we characterize the groups $\mathbf{G}_{A}$ and $\mathfrak{G}_{A}$ as well as their Lie algebras.

Let $\pm \jmath \beta_{1}, \ldots, \pm \jmath \beta_{r}$ be the distinct nonzero eigenvalues of the stable Hamiltonian matrix $A$, and let $m_{1}, \ldots, m_{r}$ be the corresponding algebraic multiplicities. Let $m_{0} \geq 0$ be the algebraic multiplicity of the zero eigenvalue $\beta_{0} \stackrel{\text { def }}{=} 0$ of $A$, so that $m_{0}+2\left(m_{1}+\cdots+m_{r}\right)=$ $2 n$. Note that $m_{0}$ is even.

For every $i=0, \ldots, r$, let $V_{i} \stackrel{\text { def }}{=}\left\{x \in \mathbb{R}^{2 n}: A^{2} x=\right.$ $\left.-\beta_{i}^{2} x\right\}$. For every $i=1, \ldots r$, the subspace $V_{i}$ is the real eigenspace of $A$ associated with the eigenvalue pair $\pm \jmath \beta_{i}$. Since $A$ is stable, each of its eigenvalues is semisimple, and hence, for every $i=1, \ldots, r$, the subspace $V_{i}$ has dimension $2 m_{i}$. The subspace $V_{0}$ is the eigenspace associated with the zero eigenvalue, and has dimension $m_{0}$. It is easy to show that each of the subspaces $V_{0}, \ldots, V_{r}$ is invariant under every element of $\mathbf{G}_{A}$ and $\mathbf{g}_{A}$.

Next, for each $i=0,1, \ldots, r$, we define $\mathbf{G}_{i} \stackrel{\text { def }}{=}\{S \in$ $\mathbf{G}_{A}: S x \in\{x,-x\}$ for every $\left.x \in V_{j}, j \neq i\right\}$ and $\mathbf{g}_{i} \stackrel{\text { def }}{=}$ $\left\{B \in \mathbf{g}_{A}: B x=0\right.$ for every $\left.x \in V_{j}, j \neq i\right\}$. In our next result, we list some properties of the groups $\mathbf{G}_{i}$ and Lie algebras $\mathbf{g}_{i}, i=0, \ldots, r$.

Proposition 5.1. The following statements hold. i) For every $i=0, \ldots, r, \mathbf{G}_{i}$ is a normal Lie subgroup of $\mathbf{G}_{A}$ with the Lie algebra $\mathbf{g}_{i}$, which is an ideal of $\mathbf{g}_{A}$. ii) $\mathbf{G}_{0}$ is homomorphic to $\operatorname{Sp}\left(\frac{1}{2} m_{0}\right)$, while, for every $i=1, \ldots, r, \mathbf{G}_{i}$ is homomorphic to the group $\left\{S \in \mathbf{S p}\left(m_{i}\right): S J_{2 m_{i}}=J_{2 m_{i}} S\right\}$.

iii) The Lie subalgebra $\mathbf{g}_{0}$ is isomorphic to the $\frac{1}{2} m_{0}\left(m_{0}+1\right)$-dimensional Lie algebra $\mathbf{s p}\left(\frac{1}{2} m_{0}\right)$, while, for every $i=1, \ldots, r, \mathbf{g}_{i}$ is isomorphic to the $m_{i}^{2}$ dimensional Lie algebra $\left\{B \in \mathbf{s p}\left(m_{i}\right): B J_{2 m_{i}}=\right.$ $\left.J_{2 m_{i}} B\right\}$.
Our next result completely characterizes the groups $\mathbf{G}_{A}$ and $\mathfrak{G}_{A}$ along with their respective Lie algebras $\mathbf{g}_{A}$ and $\mathfrak{g}_{A}$. Recall that the center of the group $\mathbf{G}_{A}$ is the subgroup $\left\{S \in \mathbf{G}_{A}: S T=T S\right.$ for every $\left.T \in \mathbf{G}_{A}\right\}$ of elements in $\mathbf{G}_{A}$ that commute with every element of $\mathbf{G}_{A}$. The proof depends on Theorem 2 of [3].

Theorem 5.1. The following statements hold. i) $\mathbf{G}_{A}=\prod_{i=0}^{r} \mathbf{G}_{i}$ and $\mathbf{g}_{A}=\mathbf{g}_{0} \oplus \cdots \oplus \mathbf{g}_{r}$. Consequently, the dimensions of the subspaces $\mathbf{g}_{A}$ and kernel $\mathcal{L}_{A}$ are each equal to $\frac{1}{2} m_{0}\left(m_{0}+1\right)+m_{1}^{2}+\cdots+m_{r}^{2}$.

ii) $\mathfrak{G}_{A}$ is the center subgroup of $\mathbf{G}_{A}$ and equals $\prod_{i \in \mathcal{K}} \mathbf{G}_{i}$, where $\mathcal{K}=\left\{i: 1 \leq i \leq r, m_{i}=1\right\}$. Moreover, $\mathfrak{g}_{A}=\bigoplus_{i \in \mathcal{K}} \mathbf{g}_{i}$. Consequently, the dimension of $\mathfrak{g}_{A}$ equals the number of simple eigenvalues of $A$.

The following corollary specializes Theorem 5.1 to the case where the matrix $A$ is simple.

Corollary 5.1. $A$ is simple if and only if $\mathfrak{G}_{A}=$ $\mathbf{G}_{A}$ if and only if $\mathbf{G}_{A}$ is commutative. If $A$ is simple, then $\mathfrak{g}_{A}=\mathbf{g}_{A}$, and $\mathbf{g}_{A}$ and kernel $\mathcal{L}_{A}$ are $n$-dimensional subspaces of $\mathbf{s p}(n)$ and $\operatorname{Sym}(n)$, respectively.

Proof. The result follows from Theorem 5.1 by noting that if $A$ is simple, then $r=n, m_{0}=0$ and $m_{i}=1$ for every $i=1, \ldots, n$, so that $\mathcal{K}=\{1, \ldots, n\}$.

\section{Undamped Mechanical Systems}

In this section, we apply Theorem 4.1 to the undamped lumped-parameter mechanical system

$$
M \ddot{q}(t)+K q(t)=0,
$$

where $q \in \mathbb{R}^{n}$, and $M, K \in \operatorname{Sym}(n)$ are the positivedefinite mass and stiffness matrices, respectively.

As a first application of Theorem 4.1, we prove the well known virial theorem which states that the timeaveraged potential and kinetic energies of the system (4) are equal. Our proof is novel in that it relies on ideas of symmetry.

Defining the state vector $y=\left[\begin{array}{ll}q^{\mathrm{T}} M^{\frac{1}{2}} & \dot{q}^{\mathrm{T}} M^{\frac{1}{2}}\end{array}\right]^{\mathrm{T}}$ yields the state space description (1) of (4) with

$$
A=\left[\begin{array}{cc}
0 & I_{n} \\
-M^{-\frac{1}{2}} K M^{-\frac{1}{2}} & 0
\end{array}\right] .
$$

The matrix $A$ is Hamiltonian. Consequently, the system (4) is Hamiltonian with the Hamiltonian function $x \mapsto x^{\mathrm{T}} H x$, where $H=-J_{2 n} A$. Under our assumptions on the mass and stiffness matrices, the system (1) is Lyapunov stable.

On letting

$$
P_{1}=\frac{1}{2}\left[\begin{array}{cc}
M^{-\frac{1}{2}} K M^{-\frac{1}{2}} & 0 \\
0 & 0
\end{array}\right], P_{2}=\frac{1}{2}\left[\begin{array}{cc}
0 & 0 \\
0 & I_{n}
\end{array}\right] \text {, }
$$


it follows that $x^{\mathrm{T}} P_{1} x=\frac{1}{2} q^{\mathrm{T}} K q$ and $x^{\mathrm{T}} P_{2} x=\frac{1}{2} \dot{q}^{\mathrm{T}} M \dot{q}$ are the potential and kinetic energies, respectively, of the system (4). It is a simple matter to verify that

$$
S=\left[\begin{array}{cc}
0 & -\left(M^{-\frac{1}{2}} K M^{-\frac{1}{2}}\right)^{-\frac{1}{2}} \\
\left(M^{-\frac{1}{2}} K M^{-\frac{1}{2}}\right)^{\frac{1}{2}} & 0
\end{array}\right]
$$

is symplectic and satisfies i) $S^{\mathrm{T}} H S=H$, so that $S \in$ $\mathbf{G}_{A}$, and ii) $S^{\mathrm{T}} P_{2} S=P_{1}$.

We claim that $S \in \mathfrak{G}_{A}$. To show this, consider $T \in \mathbf{G}_{A}$. If $T$ is partitioned as $T=\left[\begin{array}{cc}T_{1} & T_{12} \\ T_{21} & T_{2}\end{array}\right]$ where $T_{1}, T_{12}, T_{21}, T_{2} \in \mathbb{R}^{n \times n}$, then it can be shown that $T_{1}=$ $T_{2}, T_{21}=-T_{12}\left(M^{-\frac{1}{2}} K M^{-\frac{1}{2}}\right)$ and that $T_{1}, T_{12}, T_{21}, T_{2}$ commute with $M^{-\frac{1}{2}} K M^{-\frac{1}{2}}$. These facts can be used to show that $S$ commutes with $T$. Since $T \in \mathbf{G}_{A}$ was chosen arbitrarily, it follows from ii) of Theorem 5.1 that $S \in \mathfrak{G}_{A}$. Theorem 4.1 now leads to the virial theorem, which we state as the following corollary.

Corollary 6.1. The time averaged kinetic and potential energies along every solution of (4) are equal.

Our next result considers symmetries of (1) that arise from configuration-level symmetries, that is symmetries of the equation (4).

Corollary 6.2. Suppose the system (4) has distinct natural frequencies. Suppose, in addition, that there exists an orthogonal matrix $R \in \mathbb{R}^{n \times n}$ such that $R^{\mathrm{T}} K R=K$ and $R^{\mathrm{T}} M R=M$. Then, for every matrix $P \in \operatorname{Sym}(n)$, the time averages of $q^{\mathrm{T}}(t) P q(t)$ $\left(\dot{q}^{\mathrm{T}}(t) P \dot{q}(t)\right)$ and $q^{\mathrm{T}}(t) R^{\mathrm{T}} P R q(t)\left(\dot{q}^{\mathrm{T}}(t) R^{\mathrm{T}} P R \dot{q}(t)\right)$ are equal along every solution of $(4)$.

Proof. First, note that $q^{\mathrm{T}} P q=x^{\mathrm{T}} Q_{1} x$ and $\dot{q}^{\mathrm{T}} P \dot{q}=x^{\mathrm{T}} Q_{2} x$, where $Q_{1}, Q_{2} \in \operatorname{Sym}(2 n)$ are given by $Q_{1}=\left[\begin{array}{cc}M^{-\frac{1}{2}} P M^{-\frac{1}{2}} & 0 \\ 0 & 0\end{array}\right], Q_{2}=$ $\left[\begin{array}{cc}0 & 0 \\ 0 & M^{-\frac{1}{2}} P M^{-\frac{1}{2}}\end{array}\right]$. Next, consider the matrix $S=$ $\left[\begin{array}{cc}R & 0 \\ 0 & R\end{array}\right]$. It is easy to verify that $S$ is symplectic and satisfies i) $S^{\mathrm{T}} H S=H$, that is, $S \in \mathbf{G}_{A}$, and ii) $q^{\mathrm{T}} R^{\mathrm{T}} P R q=x^{\mathrm{T}} S^{\mathrm{T}} Q_{1} S x$ and $\dot{q}^{\mathrm{T}} R^{\mathrm{T}} P R \dot{q}=$ $x^{\mathrm{T}} S^{\mathrm{T}} Q_{2} S x$. Since the natural frequencies of the system are distinct, Corollary 5.1 implies that $S \in \mathfrak{G}_{A}$. The result now follows from Theorem 4.1 by letting $Q=Q_{1}$ or $Q=Q_{2}$.

If $P$ and $R^{\mathrm{T}} P R$ represent the stiffness (mass) matrices of two subsystems of (4), with $R$ a symmetry of (4), then Corollary 6.2 implies that the two subsystems will have the same potential (kinetic) energies on an average. Thus Corollary 6.2 allows us to assert that symmetrically related subsystems of the system (4) will have the same energies in the average. The following example illustrates an application of Corollary 6.2.

\section{Example 6.1.}

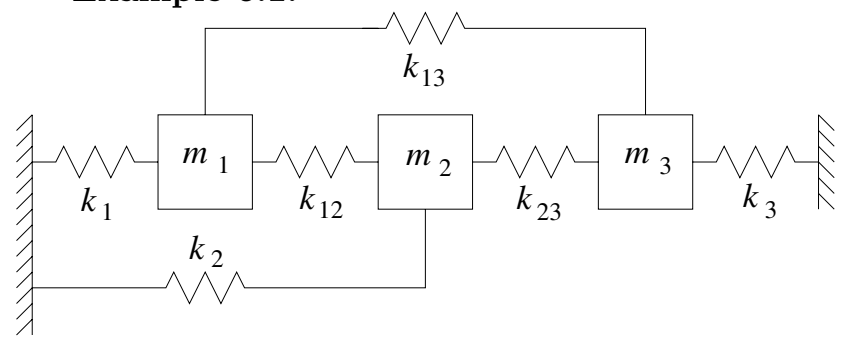

Let $M$ and $K$ denote the mass and stiffness matrices, respectively of the undamped three-degree-offreedom system depicted in the figure above. We denote the extensions in the springs $k_{1}, k_{2}$ and $k_{3}$ by $q_{1}, q_{2}$ and $q_{3}$, respectively.

Let $m_{1}=m_{2}, k_{13}=k_{23}$ and $k_{1}=k_{2}$. Then, the orthogonal permutation matrix $R$ representing the linear function $\left(q_{1}, q_{2}, q_{3}\right) \mapsto\left(q_{2}, q_{1}, q_{3}\right)$ satisfies $R^{\mathrm{T}} M R=M$ and $R^{\mathrm{T}} K R=K$. In other words, the equations (4) remain unchanged when $q_{1}$ and $q_{2}$ are interchanged.

The potential energies in the springs $k_{1}$ and $k_{2}$ are equal to $\frac{1}{2} q^{\mathrm{T}} P_{1} q$ and $\frac{1}{2} q^{\mathrm{T}} P_{2} q$, where $P_{1}=\operatorname{diag}\left(k_{1}, 0,0\right)$ and $P_{2}=\operatorname{diag}\left(0, k_{2}, 0\right)$. It is easy to verify that under our assumptions on the spring stiffnesses, $R^{\mathrm{T}} P_{1} R=$ $P_{2}$. Also, for generic values of the parameters $k_{12}$ and $k_{3}$, the system shown in the figure has distinct natural frequencies. Hence it follows from Corollary 6.2 that, generically, the average potential energy in the spring $k_{1}$ equals the average potential energy in the spring $k_{2}$ along any solution of (4).

Our next example shows that the assertion in Corollary 6.2 may not hold in the case where the system (4) has repeated natural frequencies.

Example 6.2. Consider the undamped threedegree-of-freedom system introduced in Example 6.1, with all masses and spring stiffnesses set to unity.

It is easy to verify that the equations (4) remain unchanged under orthogonal permutations that interchange $q_{1}$ with $q_{2}, q_{2}$ with $q_{3}$, and $q_{3}$ with $q_{1}$. Example 6.1 above may thus lead one to expect the potential energies in the springs $k_{1}, k_{2}$ and $k_{3}$, which transform into one another under the permutations listed above, to be equal. However, the orthogonal permutations listed above do not commute, thus indicating that the group of symmetries of the system is not commutative. There- 
fore, Corollary 5.1 implies that the system has repeated natural frequencies. Indeed, for our choice of the masses and stiffnesses, the system has only two distinct natural frequencies, namely $4 \mathrm{~s}^{-1}$ and $1 \mathrm{~s}^{-1}$, with the larger natural frequency being repeated twice. The eigenvalue associated with the repeated natural frequency, however, is semisimple (see Lemma 3 in [3]), and the system has three linearly independent mode shape vectors given by $v_{1}=\left[\begin{array}{lll}1 & -1 & 0\end{array}\right]^{\mathrm{T}}, v_{2}=\left[\begin{array}{lll}1 & 0 & -1\end{array}\right]^{\mathrm{T}}, v_{3}=\left[\begin{array}{lll}1 & 1 & 1\end{array}\right]^{\mathrm{T}}$, with $v_{1}$ and $v_{2}$ corresponding to the repeated natural frequency.

A general solution of (4) is given by $q(t)=$ $a_{1} \sin \left(4 t+\phi_{1}\right) v_{1}+a_{2} \sin \left(4 t+\phi_{2}\right) v_{2}+a_{3} \sin \left(t+\phi_{3}\right) v_{3}$, where the amplitudes $a_{1}, a_{2}$ and $a_{3}$ as well as the phases $\phi_{1}, \phi_{2}$ and $\phi_{3}$ can be assigned arbitrary values by an appropriate choice of initial conditions on $q$ and $\dot{q}$.

The average energies in the springs $k_{1}, k_{2}$ and $k_{3}$ along the general solution given above can easily be calculated to be $\frac{1}{4}\left[a_{1}^{2}+a_{2}^{2}+a_{3}^{2}+2 a_{1} a_{2} \cos \left(\phi_{1}-\phi_{2}\right)\right]$, $\frac{1}{4}\left(a_{1}^{2}+a_{3}^{2}\right)$ and $\frac{1}{4}\left(a_{2}^{2}+a_{3}^{2}\right)$, respectively. It is clear that the average energies in the springs $k_{1}, k_{2}$ and $k_{3}$ are not equal along every solution of (4). Note that unlike in Example 6.1, the average energies in the springs $k_{1}$ and $k_{2}$ are not equal along every solution, even though the symmetry that transforms the energy in $k_{1}$ to the energy in $k_{2}$ is the same as in Example 6.1. This illustrates that the assertion in Corollary 6.2 may not hold for systems having repeated natural frequencies.

\section{Conclusion}

We have explored the connections between the group of phase space symmetries of a stable linear Hamiltonian system, the set of its quadratic integrals of motion, and the operation of taking the average of a quadratic function along the trajectories of the system. Our main result is that elements of the center subgroup of the group of symmetries preserve the time averages of quadratic functions along the trajectories of the system. In the special case where the system has unrepeated eigenvalues, the group of symmetries of the system is commutative, and therefore every symmetry preserves averages of quadratic functions. These results are used to provide a novel symmetry-based proof of the virial theorem, and obtain a equipartition result for undamped linear lumped-parameter systems.

Acknowledgment: The authors thank Paolo Rapisarda and Jan Willems for providing a copy of [12].

\section{References}

[1] B. D. O. Anderson. Nonlinear networks and Onsager-Casimir reversibility. IEEE Transactions on Circuits and Systems, 27:1051-1058, 1980.
[2] V. I. Arnold. Mathematical Methods of Classical Mechanics. Springer-Verlag, New York, 1989.

[3] D. S. Bernstein and S. P. Bhat. Lyapunov stability, semistability, and asymptotic stability of matrix second-order systems. Trans. ASME, Special Combined Issue of J. Mech. Des. and J. Vibr. Acou., 117:145-153, 1995.

[4] D. S. Bernstein and S. P. Bhat. Energy equipartition and the emergence of damping in lossless systems. In Proc. IEEE Conf. Dec.and Contr., pages 2913-2918, Las Vegas, NV, 2002.

[5] R. W. Brockett and J. C. Willems. Stochastic control and the second law of thermodynamics. In Proc. IEEE Conf. Dec. and Contr., pages 10071011, San Diego, CA, 1978.

[6] M. Barahona et al. Finite horizon model reduction and the appearance of dissipation in Hamiltonian systems. In Proc. IEEE Conf. on Dec. and Contr., pages 4563-4568, Las Vegas, NV, 2002.

[7] A. Haro and R. de la Llave. New mechanisms for lack of equipartition of energy. Physics Review Letters, 85:1859-1862, 2000.

[8] Y. Kishimoto and D. S. Bernstein. Thermodynamic modeling of interconnected systems I: Conservative coupling. J. Sound Vibr., 182:23-58, 1995.

[9] Y. Kishimoto and D. S. Bernstein. Thermodynamic modeling of interconnected systems II: Dissipative coupling. J. Sound Vibr., 182:59-76, 1995.

[10] Y. Kishimoto, D. S. Bernstein, and S. R. Hall. Energy flow modeling of interconnected structures: A deterministic foundation for statistical energy analysis. J. Sound Vibr., 186:407-445, 1995.

[11] A. L. Onishchik, editor. Lie Groups and Lie Algebras I. Springer-Verlag, Berlin, 1993.

[12] P. Rapisarda and J. C. Willems. Conservedand zero-mean quadratic quantities in oscillatory systems. Technical Report M03-04, Universiteit Maastricht, 2004.

[13] J. C. Willems. Dissipative dynamical systems part I: General theory. Arch. Rat. Mech. Anal., 45:321351, 1972.

[14] J. C. Willems. Dissipative dynamical systems part II: Linear systems with quadratic supply rates. Arch. Rat. Mech. Anal., 45:352-393, 1972. 